awaited. We aim in this study to assess the expression of PDL1 using the Combined Positive Score (CPS) and to evaluate its impact on the overall survival in a cohort of 49 patients diagnosed with high-grade serous ovarian cancer.

Methods Medical charts were reviewed of 49 patients with high-grade serous ovarian cancer operated on at the gynecologic oncology department in Hôtel-Dieu de France hospital, Lebanon, between 2015 and January 2020. Immunohistochemical staining was performed for PD-L1 (Agilent Dako, PDL-1 IHC 22C3) and for TP53 (Agilent Biogenex, clone D07, 1:100 dilution) on whole tissue sections from a representative block of formalin-fixed, paraffin-embedded tumor tissue.

Results $55 \%$ of patients presented a positive PD-L1 status. No correlation was found between the PD-L1 status and the stage of the disease. Lymph node status was similar between the two cohorts, positive vs. negative CPS score ( $\mathrm{p}=0.927)$. Median follow-up was 36 months (range, 12 - 72 months). Survival rate was similar between the two cohorts, positive vs. negative PD-L1 status $(88.9 \%$ vs. $72.7 \%$ respectively, $\mathrm{p}=$ $0.14)$. No correlation was found between recurrence rate and PD-L1 status $(\mathrm{p}=0.184)$. No correlation was found between PD-L1 status and TP-53 type (wild vs. mutated) $(\mathrm{p}=0.154)$ Conclusions PD-L1 status has no impact on the prognosis of patients with high-grade serous ovarian cancer. Also, patients with TP53-mutation do not present increased expression of PD-L1 in comparison to patients with TP53 wild-type.

\section{EPV191/\#327 DRUG SCREENING OF PATIENT-DERIVED ORGANOIDS FROM OVARIAN CANCER CULTURE TO PERSONALIZED THERAPY, AN EXPLORATORY RESEARCH}

H Wang*, M He, L Zhong, D Zou. Chongqing University Cancer Hospital, The Gynecologic Oncology Center, Chongqing, China

\subsection{6/ijgc-2021-IGCS.262}

Objectives Organoids are a 3D culture model that can provide the precise genetic information and phenotype, as well as the heterogeneity of the tumor, thus provide powerful tools to model human diseases. The study (CQGOG0201) is an exploratory research to access whether organoids could guide precision treatment for OC patients.

Methods The CQGOG0201 study is a single-center, prospective, observational clinical trial. The trial design is shown in figure 1. The inclusion criteria and exclusion criteria are shown in table 1. Primary endpoint is the similarity between organoids and their corresponding tumor tissue. Secondary endpoint is the reliability of organoids obtained from IDS cases as a model for the patient's response to platinum-based adjuvant chemotherapy.

Results We completed the collection of tumor tissues from 30 different patients, including 22 HGCS patients, 3 LGCS patients, 2MC, 1 EC patients, 2 CC patients, and established

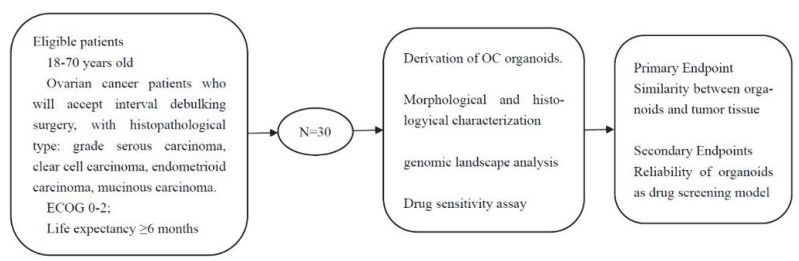

Abstract EPV191/\#327 Figure 1 Study design
9 organoid lines, derived from 15 different patients with primary tumor tissues. Organoids were derived with a success rate of $60 \%$, in particular from the HGSC, LGSC and MC
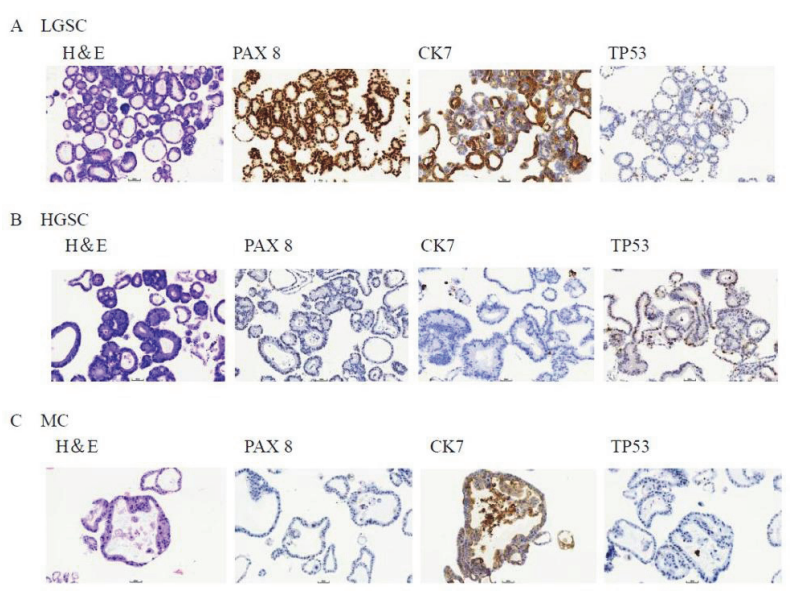

Abstract EPV191/\#327 Figure 2 Respective images of H\&E and IHC of PAX8, CK7 and TP53 from OC organoids
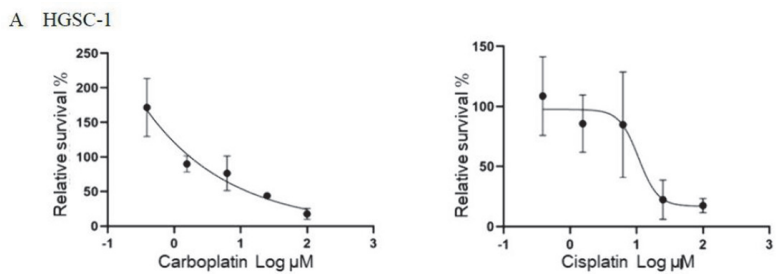

B HGCS-2
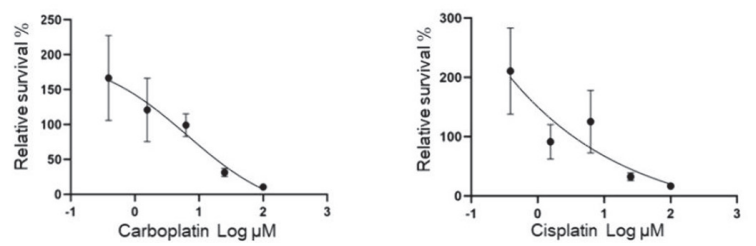

Abstract EPV191/\#327 Figure 3 Drug sensitivivty test for platinumbased chemotheraphy

Abstract EPV191/\#327 Table 1 Brief Inclusion and exclusion criteria

\begin{tabular}{|c|c|}
\hline Inclusion Criteria: & Exclusion Criteria: \\
\hline $\begin{array}{l}\text { 1. Subjects join the study voluntarily and } \\
\text { sign the informed consent; } \\
\text { 2. Ovarian cancer patients who will accept } \\
\text { primary cytoreductive surgery, with } \\
\text { histopathological type: low/high grade } \\
\text { serous carcinoma, clear cell carcinoma, } \\
\text { endometrioid carcinoma, mucinous } \\
\text { carcinoma. } \\
\text { 3. Female subjects are } 18 \text { to } 70 \text { years old; } \\
\text { 4. ECOG score } 0 \sim 2 \text {; } \\
\text { 5. Life expectancy } \geq 6 \text { months; } \\
\text { 6. Subjects have enough organ function; } \\
\text { 7. Women of child-bearing age should } \\
\text { have negative results of serum or urine } \\
\text { pregnancy test within } 7 \text { days before } \\
\text { recruited and must not be in lactation. } \\
\text { Women are willing to adopt the } \\
\text { appropriate methods of contraception } \\
\text { during the trial. }\end{array}$ & $\begin{array}{l}\text { 1. Active or uncontrolled severe infection; } \\
\text { 2. Liver cirrhosis, decompensated liver } \\
\text { disease; } \\
\text { 3. History of immune deficiency, } \\
\text { including HIV positive or suffering } \\
\text { from congenital immunodeficiency } \\
\text { disease; } \\
\text { 4. Chronic renal insufficiency or renal } \\
\text { failure; } \\
\text { 5. Any other malignant tumor has been } \\
\text { diagnosed within } 5 \text { years before } \\
\text { enrollment; } \\
\text { 6. Myocardial infarction, severe } \\
\text { arrhythmia and NYHA (New York heart } \\
\text { association) } \geq 2 \text { for congestive heart } \\
\text { failure; } \\
\text { 7. Complications that require medication, } \\
\text { which may cause severe liver and } \\
\text { kidney damage, such as tuberculosis; }\end{array}$ \\
\hline
\end{tabular}


(figure 2). OC organoids recapitulate histological features of the tumor tissues from which they were derived. In drugscreening assays, 2 organoids that were derived from HGSC patients with known clinical histories recapitulate patients' response to platinum-based adjuvant chemotherapy.

Conclusions Organoids have great potential application for research and personalized medicine. Clinical trial information: NCT04768270.

\section{EPV192/\#33 TRENDS OF CHANGE IN CANCER MORBIDITY FOR THE OVARIAN CANCER IN UZBEKISTAN}

S Djanklich*, M Tillyshaykhov, N Zakhirova, A Berkinov. Republican Specialized Scientific Practical Medical Center of Oncology and Radiology, Gynecologic Oncology Department, Tashkent, Uzbekistan

\subsection{6/ijgc-2021-IGCS.263}

Objectives Study of the trends in morbidity and mortality in ovarian cancer in the Republic of Uzbekistan from 2013 to 2017.

Methods The materials and methods of this study were the main statistical indicators for the Republic for the period 2013-2017 according to the cancer register.

Results In 2013, in the structure of the incidence rate by Uzbekistan regions the 1 st, 2nd and 3rd places were taken by Bukhara region, Tashkent city and Ferghana regions with incidence rates of 2.6, 2.2 and 2.1 per 100000 populations respectively. At the same time, in 2017 , leading positions were taken by Bukhara, Tashkent and Jizakh regions with incidence rates of 3.9, 3.3 and 3.1 per 100000 population accordingly. In 2013, there were 573 newly diagnosed cases of ovarian cancer in the Republic of Uzbekistan with incidence rate of 1.9, and 268 women died from ovarian cancer at the same year, with mortality rate of 0.9 . To compare the same indicators in 2017, it can be concluded that the rate of morbidity and mortality over the past five years had increased by 0.5 and 0.4 respectively. The percentage of patients with stages III-IV in 2013 was 67.9\%, and in 2017 this percentage decreased to $53.2 \%$.

Conclusions As can be seen from the study, over the past 5 years there have been recorded trends in the growth of morbidity and mortality in Uzbekistan. Based on this study, ovarian cancer requires more attention of oncologists in terms of timely diagnosis at the early stages of malignant growth.

\section{EPV193/\#344 SYSTEMATIC REVIEW AND META-ANALYSIS OF THE SURVIVAL IMPACT OF SECONDARY CYTOREDUCTIVE SURGERY FOR RECURRENT LOW- GRADE SEROUS OVARIAN CARCINOMA}

${ }^{1} \mathrm{R}$ Goldberg ${ }^{*},{ }^{2} \mathrm{RS}$ Kim, ${ }^{3} \mathrm{X} \mathrm{Li},{ }^{4} \mathrm{R}$ Fazelzad, ${ }^{5} \mathrm{~T}$ Brown, ${ }^{2} \mathrm{~T}$ May. ${ }^{1}$ University of Toronto, Department of Physiology, Toronto, Canada; ${ }^{2}$ Princess Margaret Cancer Centre/University of Health Network/Sinai Health Systems, Gynecologic Oncology, Toronto, Canada; ${ }^{3}$ Princess Margaret Cancer Centre, Biostatistics, Toronto, Canada; ${ }^{4}$ University Health Network, Library and Information Services, Toronto, Canada; ${ }^{5}$ Lunenfeld-Tanenbaum Research Institute, Mount Sinai Hospital, Department of Obstetrics and Gynaecology, Toronto, Canada

\subsection{6/ijgc-2021-IGCS.264}

Objectives Low-grade serous ovarian cancer (LGSC) is a relatively chemo-resistant disease with limited effective treatment options for patients with recurrence. Secondary cytoreductive surgery (SCS) is commonly offered to women with recurrent
LGSC, although the effect of cytoreductive outcomes following SCS on survival is yet to be determined. This systematic review/meta-analysis aims to evaluate the impact of SCS with gross residual disease (GRD) versus SCS with no GRD on overall survival (OS) and progression-free survival (PFS) in recurrent LGSC.

Methods A comprehensive search of MEDLINE, EMBASE, Cochrane Central, Cochrane Database of Systematic Reviews, and Web of Science was conducted to obtain all studies evaluating SCS with GRD versus no GRD in recurrent LGSC. Meta-analysis was performed on OS and PFS, and assessed using the Cochrane Risk of Bias in Non-Randomized Studies (ROBINS)-1 tool. Forest plots with pooled Hazard Ratios (HR) were generated.

Results Three retrospective cohort studies evaluating 112 LGSC patients who underwent SCS were included in the meta-analysis. Two studies were meta-analyzed for OS $(n=71)$ and PFS $(n=91)$, respectively. There were $35(31.2 \%)$ participants with no GRD at SCS, and 77 (68.8\%) participants left with GRD at SCS. GRD at SCS negatively impacted PFS $(\mathrm{HR}=3.51,95 \% \mathrm{CI}=1.72,7.14)$, and SCS with no GRD significantly improved $\mathrm{OS}(\mathrm{HR}=0.4,95 \% \mathrm{CI}=0.23,0.7)$.

Conclusions Optimal SCS with no GRD may prolong OS and PFS in women with recurrent LGSC. The quality of evidence of the included studies is low and demonstrates the need for prospective studies investigating the role of SCS in women with LGSC.

\section{EPV194/\#350 A FIVE YEARS RETROSPECTIVE REVIEW STUDY OF NON -EPITHELIAL OVARIAN CANCERS IN A THERITHERY HOSPITAL IN ETHIOPIA SUB-SHARAN COUNTRY}

B Batu*. Saint Paul's hospital Millennium Medical college, Obstetrics and Gynecology, Addis Ababa, Ethiopia

\subsection{6/ijgc-2021-IGCS.265}

Objectives The objective of this study is to describe the incidence, clinical presentation and histology subtypes and therapeutic interventions offered for NEOC.

Methods Institution based retrospective chart and pathology report review from; Aug 1 2015- Aug 1 2020. This study was conducted in Saint Paul's Hospital millennium medical college. We reviewed a total of 1357 ovarian pathology reports from the ovary in the five years period and 264 cases of which were non-epithelial ovarian tumors and of these 80 of the cases were malignant non-epithelial ovarian cancer whose pathology was retrieved and phone was accessible for interview. A preprepared structured questioner was filled by the principal investigator. The data was analyzed using IBM SPSS statistics version 20 and presented using figures and tables.

Results The contribution of malignant non-epithelial ovarian cancer is $17.3 \%$ of all the ovarian cancers. The mean age for malignant Germ cell tumors is 28.3 yrs with the range $1.75 \mathrm{yrs}$ to 61 yrs The mean age for sex cord stromal tumors is $44.5 \mathrm{yrs}$ with a range of $22 \mathrm{yrs}$ to $67 \mathrm{yrs}$ the commonest being hysterectomy, bilateral salpingo-oophrectomy with omental sampling being the commonest procedure done accounting over $40 \%$ of the cases. of those traced 7 of them are died.

Conclusions This study showed prevalence of NEOC was higher than other studies, the commonest histology type of malignant germ cell tumors was yolk sac tumors. Half of the 